\title{
Expression of a Disintegrin and Metalloprotease in Human Abdominal Aortic Aneurysms
}

\author{
Christina Lipp $^{\mathrm{a}}$ Fabian Lohoefer $^{\mathrm{a}}$ Christian Reeps $^{\mathrm{a}}$ Martina Rudelius $^{\mathrm{b}}$ \\ Markus Baummann ${ }^{c}$ Uwe Heemann ${ }^{c}$ Hans-Henning Eckstein ${ }^{a}$ \\ Jaroslav Pelisek ${ }^{a}$ \\ ${ }^{a}$ Clinic of Vascular Surgery, ${ }^{b}$ Institute of Pathology and ${ }^{\mathrm{C}}$ Department of Nephrology, Klinikum rechts der Isar der \\ Technischen Universität München, Munich, Germany
}

\section{Key Words}

Abdominal aortic aneurysm $\cdot$ A disintegrin and metalloprotease family $\cdot$ Aorta

\begin{abstract}
Objectives: The a disintegrin and metalloprotease (ADAM) family of metalloproteases possesses a proteolytic function and activates various inflammatory factors. Their expression pattern in an abdominal aortic aneurysm (AAA) is as yet unknown. The aim of this study was to make a detailed analysis of the expression of ADAMs 8, 9, 10, 12, 15 and 17, and their tissue inhibitors of metalloprotease (TIMP)- 1 and TIMP-3 in patients with AAA. Design: The aortic vessel walls of AAA patients $(n=20)$ and non-aneurysmal aortic specimens $(n=$ 10) were obtained by conventional surgical repair and autopsy. SYBR green-based real-time PCR, histology and immunohistochemistry were performed on all samples. Main Outcome Measures: Quantitative expression analysis and the localisation of various ADAMs in AAA. Results: ADAMs tested in our study were expressed in both AAA and control aorta without any significant differences between the groups. In contrast, expression of TIMP-1 was significantly reduced in AAA compared to control vessels. Smooth mus-
\end{abstract}

cle cells (SMCs), neovessels and macrophages were positive for all ADAMs and TIMPs tested. Infiltrates were negative for TIMP-3, and luminal endothelial cells were positive for ADAMs 15 and 17. A significant positive correlation was observed between ADAMs 10, 12, 15, 17, TIMP-3 and SMCs. Conclusion: ADAMs are constitutively expressed in normal aortic vessel walls and AAA, particularly in SMCs.

Copyright $\odot 2012$ S. Karger AG, Base

\section{Introduction}

Despite considerable advances in surgical treatment, the ruptured human abdominal aortic aneurysm (AAA) is still associated with a mortality rate of $65-85 \%[1,2]$. A diameter is currently the only reliable determinant of the imminent rupture of an AAA and patients exceeding 5.5 $\mathrm{cm}$ generally undergo surgical or endovascular intervention $[2,3]$. However, the outcome of rupture is poor with less than half of the patients being delivered to the hospital alive $[1,4]$.

It is well known that inflammation and proteolytic degradation markedly contributes to the formation and rupture of an AAA [5, 6]. During inflammation, the aor-

\section{KARGER}

Fax +4161306 1234

E-Mail karger@karger.ch

www.karger.com (c) 2012 S. Karger AG, Basel

$1018-1172 / 12 / 0493-0198 \$ 38.00 / 0$

Accessible online at:

www.karger.com/jvr
Prof. Dr. med. Hans-Henning Eckstein

Department of Vascular Surgery

Klinikum rechts der Isar der Technischen Universität München

Ismaninger Strasse 22, DE-81675 Munich (Germany)

Tel. +49 894140 2167, E-Mail gefaesschirurgie@lrz.tum.de 
tic wall is weakened by loss of smooth muscle cells (SMCs) and destruction of the extracellular matrix (ECM), especially elastic fibres [5]. The responsibility of proteolytic enzymes, such as matrix metalloproteinases (MMPs), in the degradation of the aortic wall has already been well examined [7-9]. The role of other subgroups of proteolytic enzymes with a disintegrin and metalloprotease (ADAMs) domain in AAA is, however, unknown. Besides their proteolytic function, most ADAMs are able to process and activate other proteins and enzymes [10-20]. For example, ADAMs 10 and 17 catalyse the shedding of tumour necrosis factor (TNF)- $\alpha$, which is an important pro-inflammatory enzyme [11, 12]. Furthermore, ADAMs are involved in the shedding of various ligands of epidermal growth factor receptors, e.g. heparin-binding epidermal growth factor and transforming growth factor- $\alpha$ [12]. There is also evidence that epidermal growth factor ligands are involved in the pathogenesis of atherosclerosis [13]. Furthermore, some studies have described increased expression of ADAMs in connection with malign tumours $[11,14]$. ADAMs 10,12 and 15 are able to cleave components of the ECM and vascular wall such as collagen IV or gelatine $[11,15,16]$. However, hitherto only little is known about the function of ADAMs in atherosclerosis and even less about their presence and possible role in AAA. ADAM-15 has already been detected in vascular SMCs of atherosclerotic vessels but interestingly not in SMCs of control arteries [17]. Hence, another study described increased expression of ADAM-17, also known as TACE (TNF- $\alpha$-converting enzyme), in human AAA [18]. Especially in the transition zone, high levels of ADAM-17 and TNF- $\alpha$ have been detected, implementing a certain role in the development of AAA.

Therefore, the aim of the present study was to conduct an expression analysis of ADAMs with proteolytic function [19] such as ADAMs 8, 9, 10, 12, 15 and 17, and their tissue inhibitors of metalloprotease (TIMP)-1 and TIMP3 [20] in human AAA by quantitative PCR. The localisation of the corresponding ADAMs was determined by means of immunohistochemistry.

\section{Patients and Methods}

\section{Patient Demographics}

Samples of 20 patients were collected in a standardised manner from the aneurysmal body during the open surgical procedure of AAA. The tissue samples were segmented in blocks of 3-4 mm (3-5 blocks), immediately fixed in formalin and after $24 \mathrm{~h}$ embedded in paraffin. The average age of the patients was $67.7 \pm 12.6$ years (17 male, 3 female). The mean diameter of aneurysm detected was $7.2 \pm 1.9 \mathrm{~cm}$. Control samples of 10 non- aneurysmal infrarenal abdominal aortas were obtained within 1 day of death from the Institute of Forensic Pathology of the University Munich. The quality of RNA and the integrity of cDNA were proven by an RNA/cDNA Quality Assay Kit (Clontech, Heidelberg, Germany). None of the control samples showed largescale atherosclerotic lesions or suffered from connective tissue disease, e.g. Ehlers-Danlos syndrome.

The study was conducted with the approval of our institutional review board and ethics commission. Informed consent was obtained from all patients prior to their participation in the study. The investigation conformed to the principles outlined by the Declaration of Helsinki for use of human tissue or subjects [21].

\section{Quantitative Real-Time PCR}

Quantitative real-time PCR was performed with specimens from AAAs and control aortas fixed in formalin and embedded in paraffin. The material for real-time PCR was obtained from the same paraffin blocks used for histological examination. It was obtained subsequently as a consecutive tissue material to the histological slides. The RNA was isolated using the High Pure RNA Paraffin Kit according to the manufacturer's instructions (Roche, Mannheim, Germany) and further modified in our laboratory in order to increase the total yield of RNA. The isolated RNA was transcribed into cDNA using the cDNA Synthesis Kit RevertAid (Fermentas, St. Leon-Rot, Germany). Quantitative real-time PCR was performed using SYBR Green fluorescence dye (peqLab, Erlangen, Germany) and SYBR Green Cycler iQ ${ }^{\mathrm{TM}} 5$ real-time PCR Detection System (Bio-Rad, Hercules, Calif., USA) at the following conditions: initialization step $5 \mathrm{~min}$ at $95^{\circ} \mathrm{C}$; denaturation $10 \mathrm{~s}$ at $95^{\circ} \mathrm{C}$; annealing $30 \mathrm{~s}$ at $55^{\circ} \mathrm{C}$ (ADAM-9), $60^{\circ} \mathrm{C}$ (ADAMs 8,10 , $12,15,17$, TIMP-1 and TIMP-3) or $62^{\circ} \mathrm{C}$ (glyceraldehyde 3 -phosphate dehydrogenase, GAPDH); extension $10 \mathrm{~s}$ at $72^{\circ} \mathrm{C}, 40$ cycles. All results were normalised for the expression of GAPDH. Furthermore, the PCR results were proven by gel electrophoresis. The following primers were applied in the present study: GAPDH forward 5'-CACTGCCAACGTGTCAGTGGTG-3', reverse 5'-TGTAGCCCAGGATGCCCTTGAG-3'; ADAM-8 forward 5'-CCCAGCTTTGTGTGTGTTTAAG-3', reverse 5'-AATAAAAGCTTTCAGCCCTGCT-3'; ADAM-9 forward 5'-TCAGAGGATTGCTGCATTTAGA-3', reverse 5'-CTCCACATTTCAGAGGCTCTTT-3'; ADAM-10 forward 5'-ACCCTACAAATCCTTTCCGTTT-3', reverse 5'-CATCAAAATCTCGGTCTGTGAA-3'; ADAM12 forward $5^{\prime}$-CCTTAGATCTGGCATGTTAGCC-3', reverse 5' ATCAAACTCTCCTGGCTCTCTG-3'; ADAM-15 forward $5^{\prime}$ GACAGTGTCCTCGCTCTACCTC-3', reverse 5' -CTTCAGTCATGGTAGGGGACTC-3'; ADAM-17 forward 5'-GGGACATAATTTTGGAGCAGAA-3', reverse 5'-CCACTCACAGCTATGGGATACA-3'; TIMP-1 forward 5'-GAAAAGGGCTTC-CAGTCCCGTC-3', reverse 5'-ATTCAGGCTATCTGGGACCGCA-3'; TIMP-3 forward 5'-CTTCCCAGATGATGACAATGAA-3', reverse 5'-ACCCCATATGACAGCATAGACC-3'.

\section{Histology and Immunohistochemistry}

Sections of 2-3 $\mu \mathrm{m}$ were cut from each segment of the formalin-fixed paraffin-embedded aortic tissue samples. Aortic tissues were removed from a central part of the aneurysmal body, segmented into $3-5$ blocks dependent upon the sample size and treated accordingly. In order to create a series of repetitive slides, the same paraffin block was used for all examinations. First, haemalum-eosin (HE) and elastica-van Gieson (EvG) staining was 
performed in order to assess the sample morphology, cellularity and degradation of the aortic vessel wall. For immunohistochemistry, dewaxed and hydrated sections were boiled in citric buffer ( $\mathrm{pH}$ 6.0) to retrieve the epitopes of antigens, washed and treated with appropriate antibodies.

For cell characterisation, AAA samples and control aortic tissues were treated with antibodies against vascular SMCs (antismooth muscle actin, mouse monoclonal, clone HHF35, dilution 1:200; Dako, Glostrup, Denmark), endothelial cells (anti-von Willebrand factor, mouse monoclonal, clone F8/86, dilution 1:500; Dako), macrophages/monocytes (anti-CD68, mouse monoclonal, clone KP1, dilution 1:2,000; Dako), T and B lymphocytes (anti-CD3, rabbit polyclonal, dilution 1:400 and anti-CD20, mouse monoclonal, clone L26, dilution 1:500; Dako). For detection of ADAMs and TIMPs, the following antibodies were applied: anti-ADAM-8 (goat polyclonal, dilution 1:25; R\&D Systems), anti-ADAM-10 (rabbit polyclonal, dilution 1:30; abcam, Cambridge, UK), anti-ADAM-15 (mouse monoclonal, dilution 1:50; R\&D Systems), anti-ADAM-17 (rabbit polyclonal; dilution 1:800; R\&D Systems), anti-TIMP-1 (rabbit polyclonal, dilution 1:100; Lab Vision), and anti-TIMP-3 (mouse monoclonal, dilution $1: 100$; abcam).

Following primary antibody incubation, von Willebrand factor and smooth muscle actin were visualized using the APAAP ChemMate Detection Kit (rabbit anti-mouse; Dako) according to the manufacturer's instructions. All other primary antibodies were detected by LSAB ChemMate Detection Kit (biotinylated goat anti-mouse/anti-rabbit; Dako).

\section{Histological and Statistical Evaluation}

The intensity of staining was evaluated in all specimens prior to the validation of the corresponding intensities within each sample. The graduation was performed via light microscopy by two experienced investigators blinded for the study samples applying the following semiquantitative graduation: no staining $(-)$; positive staining of some $(+)$ or most $(++)$ cells and the majority of specimens; strong overall positive staining detected in all cells and all specimens $(+++)$, and the intensity of staining varied between different samples and also within the same specimen $(-/+)$.

All results were analysed using SPSS for Windows version 17.0. (SPSS Inc., Chicago, Ill., USA). Values of continuous variables were compared using non-parametric Mann-Whitney U test. Statistical differences between more than two groups were analysed by non-parametric Kruskal-Wallis test. The data were presented by a box plot diagram showing the median and the 25 th and 75 th percentile. Correlations between continuous variables were quantified using Pearson's correlation coefficient for normal distributed samples or by Spearman's rank correlation coefficient. All statistical comparisons were performed two sided in the sense of an exploratory data analysis using $<0.05$ level of significance.

\section{Results}

Quantitative Expression Analysis

Using formalin-fixed paraffin-embedded tissue samples, the expression of all ADAMs and their inhibitors TIMP-1 and TIMP-3 analysed in our study was detected in both AAA tissue samples and control aorta. Standard normalisation of the quantitative PCR values to GAPDH demonstrated no significant differences in the expression of ADAMs between the study groups (fig. 1). Interestingly, there was even a tendency of higher expression of ADAMs in the control vessels compared to the AAA samples. The same expression pattern was also found for TIMP-3. Significant differences were observed only for TIMP-1 ( $p=0.019$ ). Furthermore, to obtain an overview of the expression levels of all ADAMs and their inhibitors, the expression data at mRNA level are summarised in figure 2. TIMP-1 demonstrated the highest expression in the control aortic tissue. In contrast, the expression of TIMP-3 was about 11-fold lower compared to TIMP-1 in both the control tissue and AAA samples. Regarding ADAMs, the highest expression was observed for ADAMs 10, 12, and 15. The lowest expression was observed for ADAM-9 with about a 50-fold lower level of mRNA in AAA tissue samples and about a 200-fold lower level of mRNA in aortic controls.

\section{Histological Analysis and Immunohistochemistry}

To associate the expression of ADAMs with the different cell types localised in AAA, histological and immunohistochemical analyses of ADAMs, TIMP-1 and TIMP-3 were performed. First, AAA samples were characterised by using HE and EvG staining. Tissue samples were stained with CD68 for macrophages, smooth muscle actin for SMCs and von Willebrand factor for endothelial cells (ECs; table 1). Neovascularisation was mainly localised in the area of the deeper part of the media distant from the lumen or between media and adventitia and was mainly associated with inflammatory infiltrates. Widespread loss of elastin, collagen and SMCs was observed in all AAA tissue samples. Most AAA samples were atherosclerotic with extended calcified areas. AAA samples were further stained with antibodies against ADAMs 8, 10, 15 and 17 and also against TIMP-1 and TIMP-3 (fig. 3). The expression of ADAM-9 at mRNA level was very low and therefore we did not perform any immunohistochemistry for this metalloproteinase. Furthermore, we did not find a suitable antibody for ADAM-12 and so the staining against this ADAM was also omitted. The staining of the luminal endothelial cells was negative for all ADAMs and TIMPs tested, with the exception of ADAMs 15 and 17 (data not shown). In contrast, medial SMCs, macrophages and inflammatory cells were positive for all ADAMs tested and for TIMP-1 and TIMP-3 (fig. 3a, b). Furthermore, neovessels expressed ADAMs 8, 10, 15 and 17, and TIMP1 and TIMP-3 (fig. 3c). Regarding the individual ADAMs, 


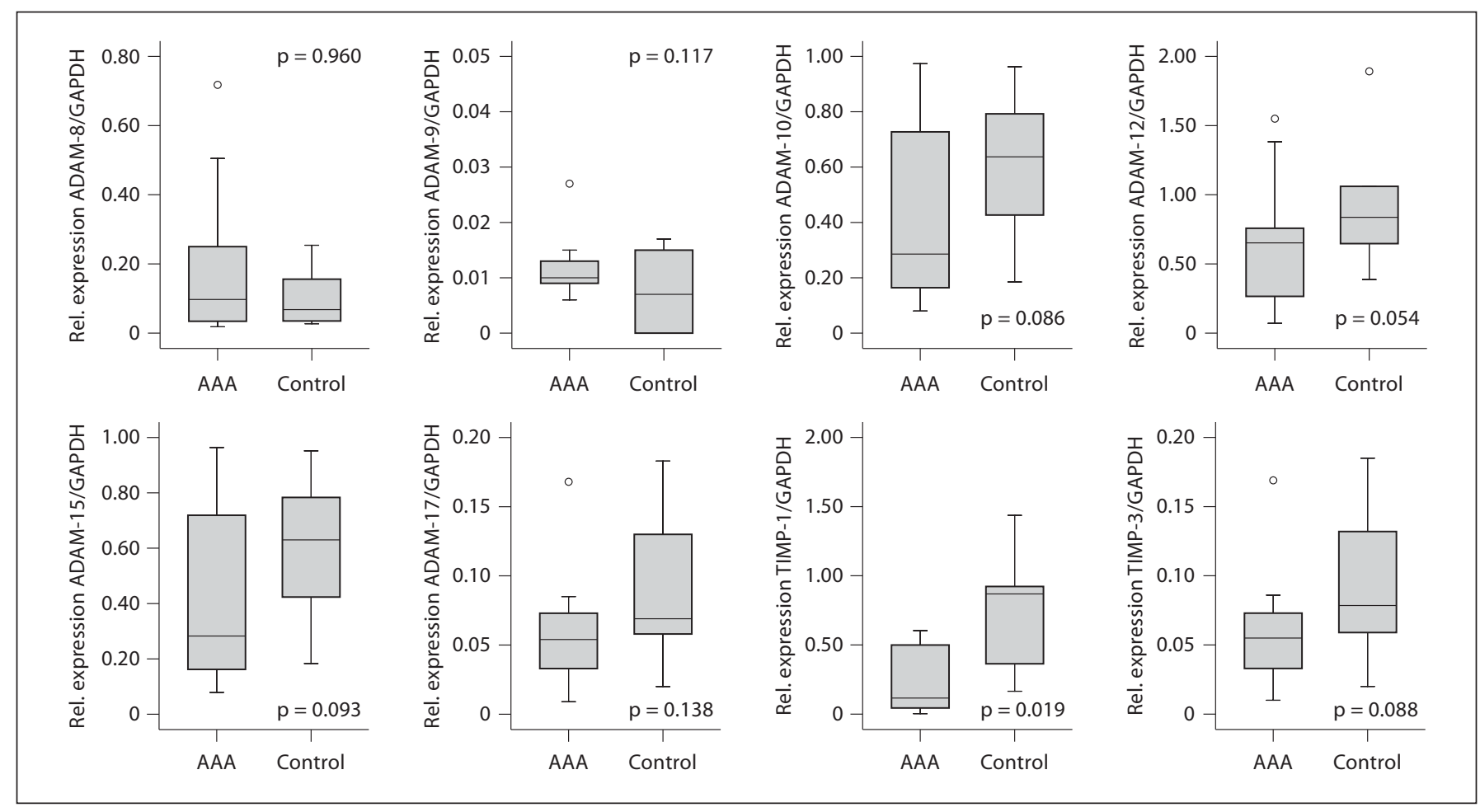

Fig. 1. Expression of ADAMs 8, 9, 10, 12, 15 and 17, TIMP-1 and TIMP-3 in AAA tissue samples compared to control healthy aorta, analysed by quantitative real-time PCR and SYBR Green fluorescence dye. Expression of the single ADAMs on an mRNA level was normalised against GAPDH.

ADAMs 8 and 10 seemed to be expressed mainly in macrophages, infiltrates and neovessels, and ADAMs 15 and 17 in addition also in SMCs. TIMP-1 was detected particularly in macrophages and inflammatory cells, and TIMP-3 mainly in macrophages.

\section{Correlation Analysis}

For better understanding of the behaviour of ADAMs in the aortic vessel wall, expression of these proteinases was correlated with specimen morphology (content of elastin and collagen, macrophages, SMCs, inflammation, neovascularisation) and clinical occurrence of AAA. Significant correlation was observed between ADAMs 10, 12,15 and 17, TIMP-3 and the content of SMCs within the aneurysmal vessel wall $(\mathrm{r}=0.486,0.511,0.478,0.589$ and 0.590 , respectively; $\mathrm{p}<0.05)$. No significant correlations were observed between the expression of ADAMs or TIMPs and other vessel wall components. Regarding clinical parameters, positive correlation was observed for ADAM-17, TIMP-3 and CRP ( $\mathrm{r}=0.471$ and 0.586; $\mathrm{p}<$ $0.05)$, and negative correlation for TIMP-1 and CRP ( $\mathrm{r}=$ $-0.572 ; \mathrm{p}<0.05)$. In addition, to understand the inter-

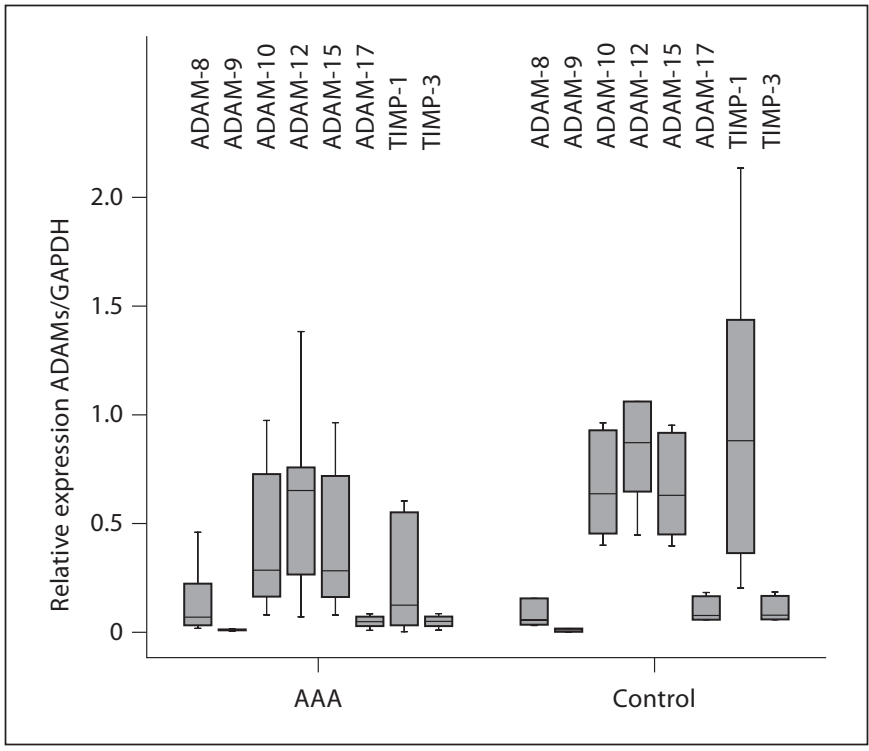

Fig. 2. An overview: expression of ADAMs 8, 9, 10, 12, 15 and 17, TIMP-1 and TIMP-3 in AAA tissue samples compared to control non-aneurysmal aortic tissues, analysed by quantitative real-time PCR and SYBR Green fluorescence dye as shown in figure 1, normalised against the expression of GAPDH. 


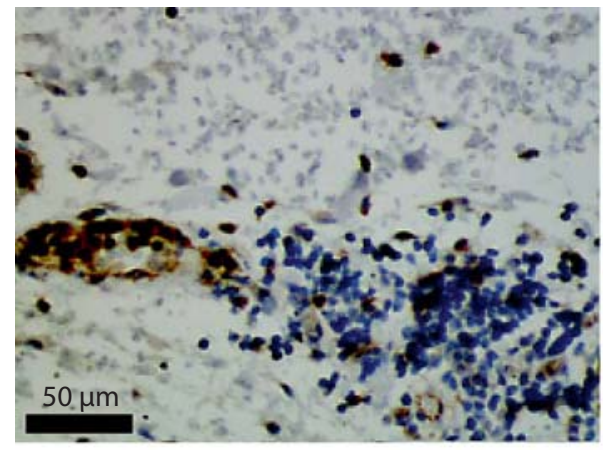

ADAM-8

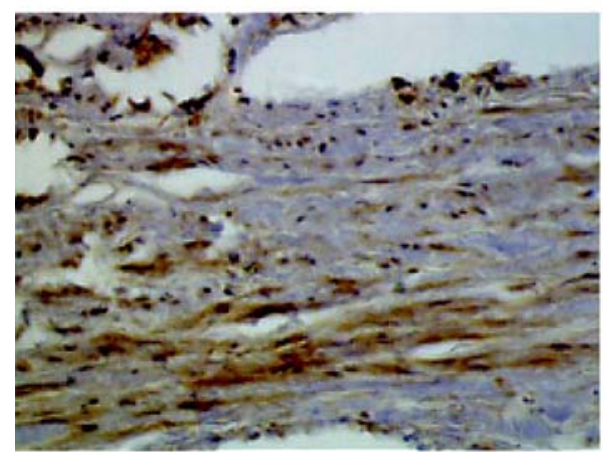

ADAM-15

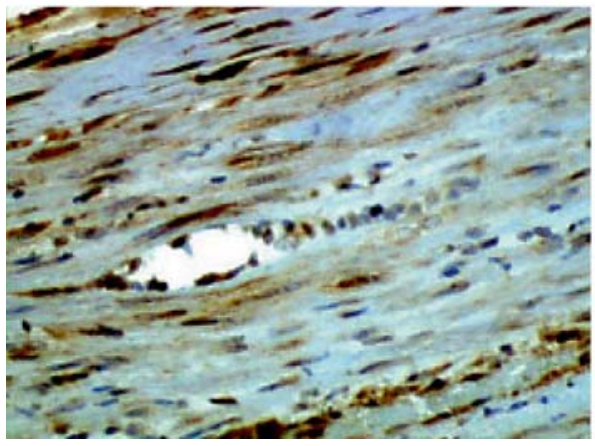

TIMP-1

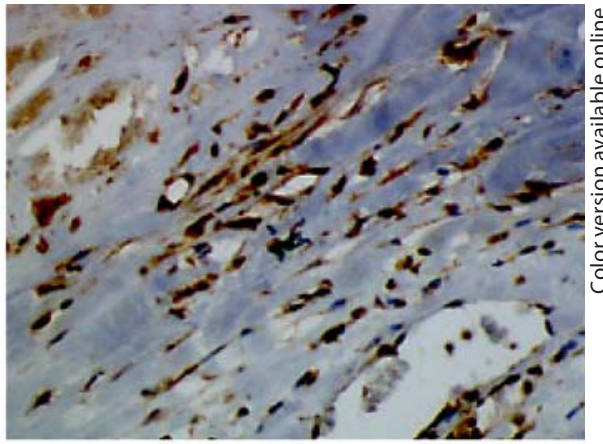

ADAM-10

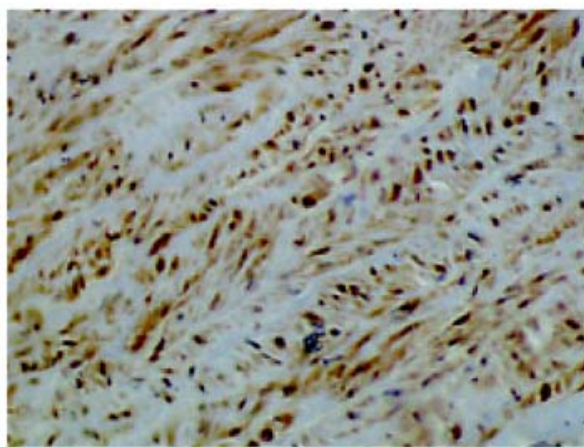

ADAM-17

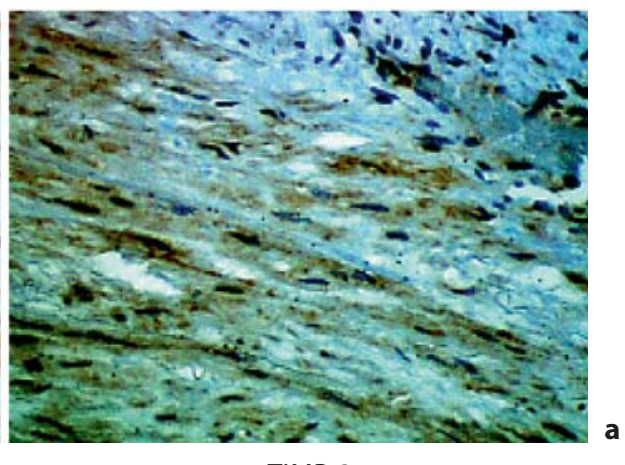

TIMP-3 a tures.

Fig. 3. Selective immunohistochemical staining of ADAMs 8, 10, 15 and 17, TIMP and TIMP-3 in smooth muscle cells (a) inflammatory cells (b) and neovessels (c) within human AAA tissues samples. The staining of ADAMs and TIMPs is brown, cells are counterstained with haemalum eosin (blue; colours online version only). The scale bar in the first picture (upper

Table 1. Histological and morphological characteristics of tissue samples of AAA study patients

\begin{tabular}{|c|c|c|c|c|c|c|c|c|}
\hline & Cellularity ${ }^{1}$ & Infiltrates & Elastin & Collagen & Macrophages & SMCs & Neovessels & Calcification \\
\hline Patients $(n=20)$ & $+/++$ & ++ & + & $+/++$ & $+/++$ & + & $+/++$ & + \\
\hline Range (min, max) &,++++ & $+/-,+++$ &,-++ &,+++ & $+/-,+++$ & $+/-,+++$ & $+1-,+++$ &,-+++ \\
\hline
\end{tabular}

\footnotetext{
${ }^{1}$ Cellularity means the overall density of cells within the sample-inclusive infiltrates.

${ }^{2}$ For all control aortic tissue samples the results were the same.

${ }^{3}$ Small vessels are found at the border between media and adventitia. These microvessels are, however, not associated with any infiltrates and contribute generally to the blood supply of the normal large vessels.
} 


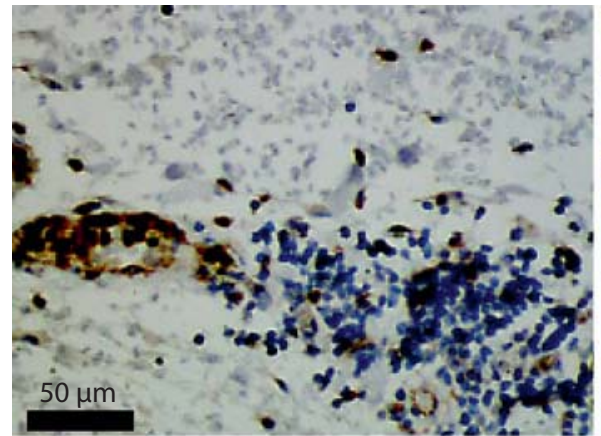

ADAM-8

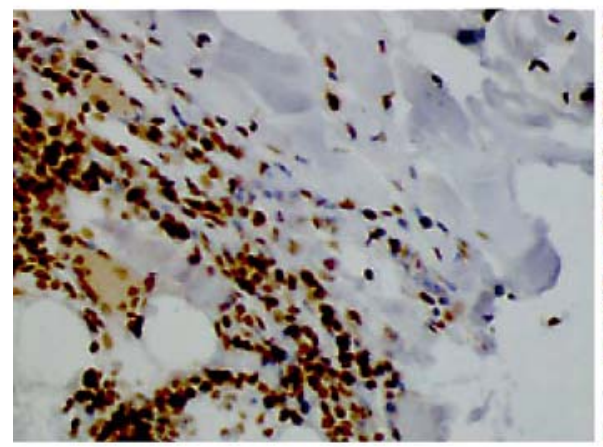

ADAM-15

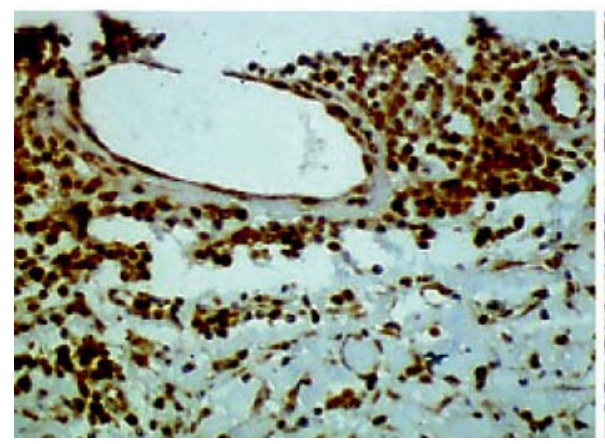

TIMP-1

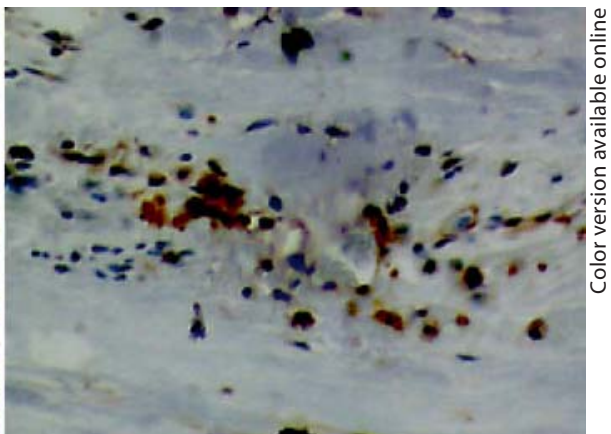

ADAM-10

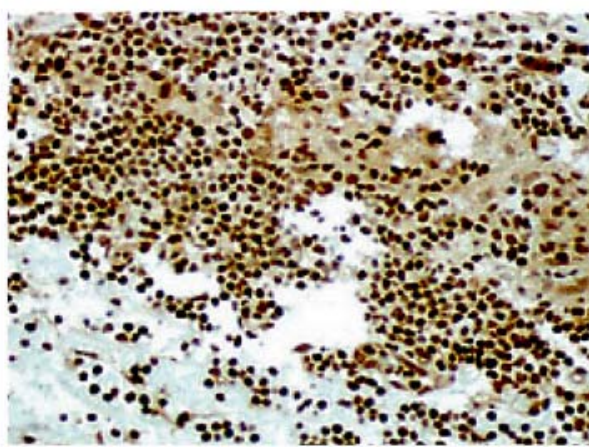

ADAM-17

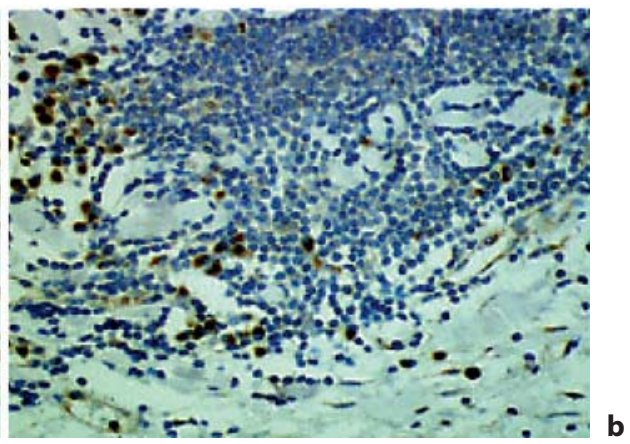

TIMP-3 tures.

Fig. 3. Selective immunohistochemical staining of ADAMs 8, 10, 15 and 17, TIMP inflammatory cells (b) and neovessels (c) within human AAA tissues samples. The staining of ADAMs and TIMPs is brown, cells are counterstained with haemalum eosin (blue; colours online version only). The scale bar in the first picture (upper

related relationships between the single ADAMs and their inhibitors, their expression was compared to each other. Interestingly, with the exception of ADAM-8 and TIMP-1, all other ADAMs and also TIMP-3 correlated significantly to each other $(r>0.700 ; p<0.001)$. Thus, the expression of ADAMs and TIMP-3 seems to be regulated by the same factors. ADAM-9 correlated with ADAMs $10,12$ and 17 ( $\mathrm{p}<0.05)$, and ADAM-8 with ADAMs 10 and $15(\mathrm{p}<0.05)$. No correlation was observed between any single ADAM and TIMP-1.

Considering that mRNA-based expression analysis does not provide concrete information on protein expres- sion and activity, we attempted to normalise ADAMs on their relevant inhibitors (TIMPs) instead of GAPDH. These data indicated significant increased activity of ADAMs 10 and 15 in AAA.

\section{Discussion}

The development of AAA is closely associated with alternation of connective tissue in the aortic wall, especially fragmentation of elastic fibres and collagen degradation through various proteolytic enzymes, particularly 


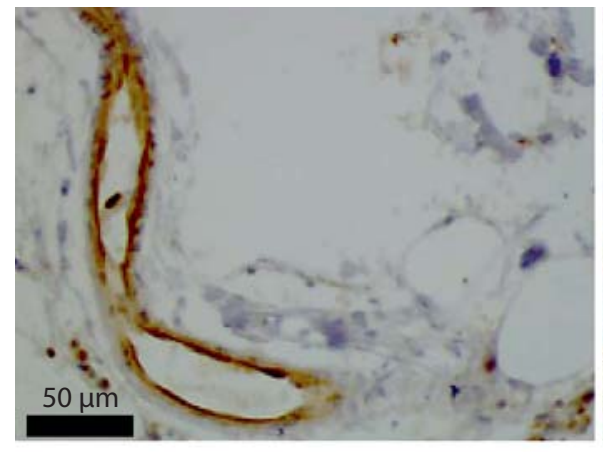

ADAM-8

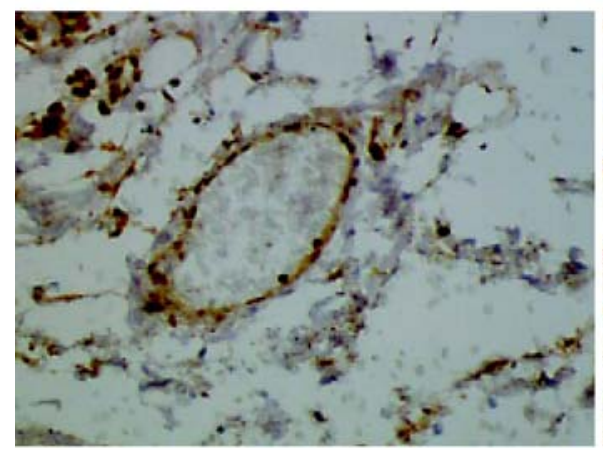

ADAM-15

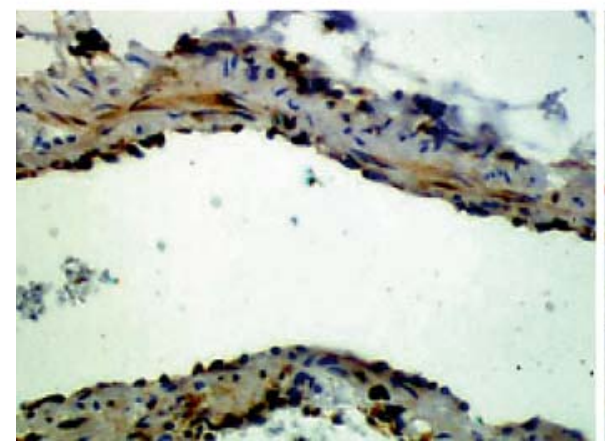

TIMP-1

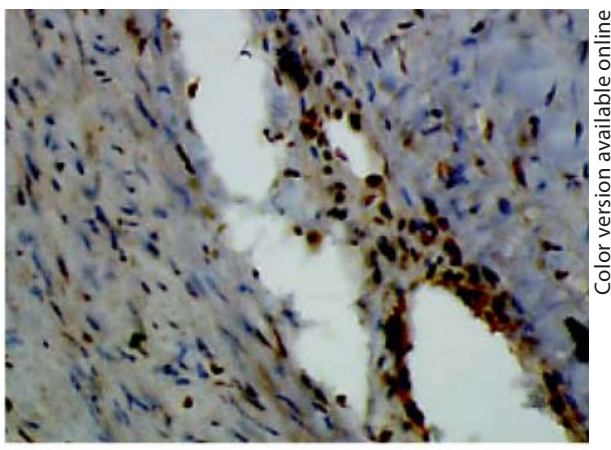

ADAM-10

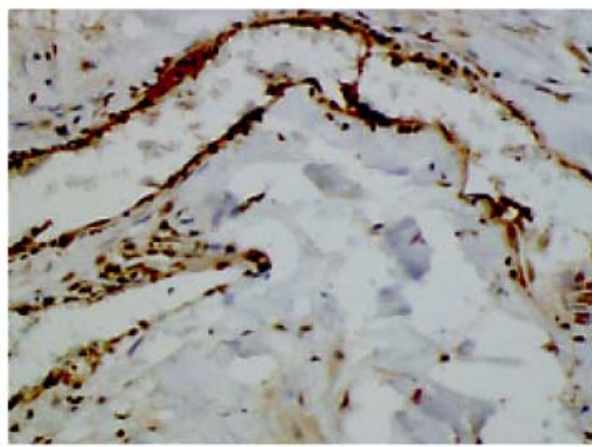

ADAM-17

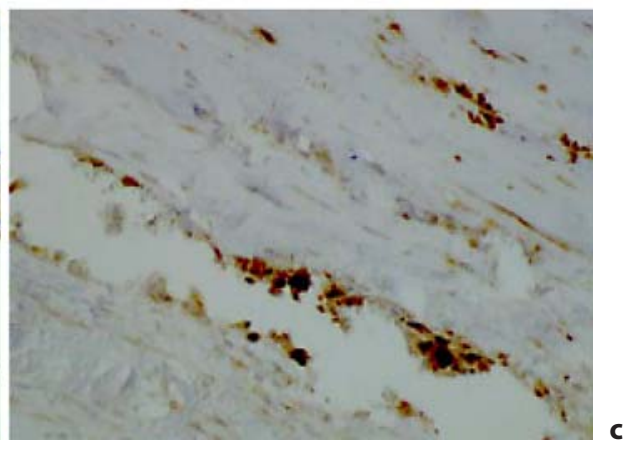

TIMP-3 tures.

MMPs $[8,9,22]$. Some promising drugs inhibiting MMPs have already been shown to be successful in experimental models [23]. However, no positive results have been observed in clinical trials. This phenomenon may be based on the fact that MMPs are not the only proteinases able to degrade connective tissue. There is a plethora of proteolytic enzymes belonging to the family of metalloproteinases such as ADAMs with multiple biological roles including cell-matrix interaction, zymogen activation (shedding) and cell adhesion. These processes contribute to cell migration, proteolysis and angiogenesis [24]. The effect of ADAMs on the pathology of AAA is as yet unknown, although some researchers have proposed the role of ADAMs 9, 15 or 17 in atherosclerosis [25-28]. No recent studies have been performed in human AAAs with the exception of ADAM-17 $[9,24]$.

Our results demonstrate that all ADAMs known for their proteolytic activity such as ADAMs 8, 9, 10, 12, 15 and 17 are expressed in both AAA samples and control aortic tissues. Surprisingly, regarding the absolute expression of ADAMs at mRNA level, it was either similar for both study groups or even higher in the control group. These results were partially in discordance with the data by Herren et al. [17], who observed no expression of ADAM-15 in control vessels, for example. However, in contrast to our study where we analysed the expression of 
ADAMs directly in the aortic tissue samples, those researchers used explanted human aortic SMCs in cell culture, the conditions of which may generate significantly different results compared to tissue samples. A healthy aortic vessel wall is composed mainly of SMCs and consequently ADAMs are expressed predominantly in these cells. The results were further confirmed by our correlation analysis. The expression of all ADAMs correlated significantly with the amount of SMCs within the aortic vessel with the exception of ADAMs 8 and 9. In contrast to the study by Oksala et al. [24], we did not observe any correlation between the appearance of macrophages or inflammatory cells within AAA and expression of the analysed ADAMs. There was also no significant correlation between the frequently observed extent of neovascularisation and ADAMs expression. So far, only one study has described the expression of ADAMs in AAA. Satoh et al. [18] detected elevated levels of ADAM-17 mRNA in AAAs compared to a control. In contrast, we did not observe any differences in the expression of ADAM-17 between AAA and healthy aorta.

Regarding the localisation of ADAMs expression within the aortic vessel wall by means of immunohistochemistry, SMCs were, as expected, positive for all ADAMs tested and are probably the main source of ADAMs in a healthy aorta. Interestingly, Satoh et al. [18] located ADAM-17 mainly in macrophages. In AAA, inflammatory cells and neovessels were also positive for all ADAMs tested in our study. Thus, ADAMs seem to be of particular relevance to the cellular activity, proliferation and migration also in the AAA wall, contributing to atherosclerosis and degradation of the ECM [26]. ADAMs mediate proteoglycan and integrin bindings, cell-cell and cell-matrix interaction in the vessel wall $[17,20,27,28]$.

The activity of proteolytic enzymes is closely associated with the expression of their corresponding inhibitors [11]. Control aortic tissues in our experiments ex- pressed significantly more TIMP-1 than AAA tissues. The expression of TIMP-3 was also increased, however, without the postulated statistical significance. Therefore, we performed an additional normalisation of the expression of ADAMs against the expression of their inhibitors $[19,20]$. Interestingly, following this, significant higher activities were observed for ADAMs 10 and 15 compared to control aorta. However, it is of note that normalisation upon the basis of mRNA-based expression does not provide any information on enzyme activity due to the different mRNA and protein stability. Therefore, these data have to be further validated on a protein level. Furthermore, even if we proved the quality of RNA isolated from the formalin-fixed samples, we cannot exclude damage in the transcripts of the individual ADAMs tested in our study following formalin treatment. Thus, our results have to be verified in further studies.

\section{Conclusions}

Our results conclude that ADAMs 8, 9, 10, 12, 15 and 17 are constitutively expressed in aortic vessel walls, particularly in SMCs. Their expression might be necessary for normal cellular function such as cell-cell and cell-matrix interaction, degradation of the ECM, migration and activation of proteolytic enzymes, and various signal molecules [29]. In contrast to other studies, our results did not confirm any upregulation of ADAMs in AAA. Further studies are necessary to better understand their function in the vessel wall.

\section{Acknowledgements}

This work was supported by the Dr. Robert Pfleger Stiftung. The authors thank Renate Hegenloh for her technical competence and help.

\section{References}

1 Eckstein H, Böckler D, Flessenkämper I, Schmitz-Rixen T, Debus S, Lang W: Ultrasonographic screening for the detection of abdominal aortic aneurysms. Dtsch Ärztebl Int 2009;106:657-663.

2 Sakalihasan N, Limet R, Defawe OD: Abdominal aortic aneurysm. Lancet 2005;365: 1577-1589.

3 Lederle FA: In the clinic: abdominal aortic aneurysm. Ann Intern Med 2009;150:ITC51-ITC5-15.

Expression of ADAM Proteases in AAA
4 Golledge J, Muller J, Daugherty A, Norman P: Abdominal aortic aneurysm: pathogenesis and implications for management. Arterioscler Thromb Vasc Biol 2006;26:26052613.

5 Guo DC, Papke CL, He R, Milewicz DM: Pathogenesis of thoracic and abdominal aortic aneurysms. Ann NY Acad Sci 2006;1085: 339-352.

6 Herron GS, Unemori E, Wong M, Rapp JH, Hibbs MH, Stoney RJ: Connective tissue proteinases and inhibitors in abdominal aortic aneurysms: involvement of the vasa vasorum in the pathogenesis of aortic aneurysms. Arterioscler Thromb 1991;11:16671677.

7 Pearce WH, Shively VP: Abdominal aortic aneurysm as a complex multifactorial disease: interactions of polymorphisms of inflammatory genes, features of autoimmunity, and current status of MMPs. Ann NY Acad Sci 2006; 1085:117-132. 
$>8$ Keeling WB, Armstrong PA, Stone PA, Bandyk DF, Shames ML: An overview of matrix metalloproteinases in the pathogenesis and treatment of abdominal aortic aneurysms. Vasc Endovasc Surg 2005;39:457-464.

$>9$ Galis ZS, Sukhova GK, Lark MW, Libby P: Increased expression of matrix metalloproteinases and matrix degrading activity in vulnerable regions of human atherosclerotic plaques. J Clin Invest 1994;94:2493-2503.

$\checkmark 10$ Blobel CP: ADAMs: key components in EGFR signalling and development. Nature 2005;6:32-43.

11 Seals DF, Courtneidge SA: The ADAMs family of metalloproteaes: multidomain proteins with multiple functions. Genes Dev 2003;17: 7-30.

12 Sahin U, Weskamp G, Kelly K, Zhou HM, Higashiyama S, Peschon J, et al: Distinct roles for ADAM 10 and ADAM 17 in ectodomain shedding of six EGFR ligands. J Cell Biol 2004; 164:769-779.

13 Dreux AC, Lamb DJ, Modjtahedi H, Ferns GA: The epidermal growth factor receptors and their family of ligands: their putative role in atherogenesis. Atherosclerosis 2006; 186:38-53.

14 van Goor H, Melenhorst WB, Turner AJ, Holgate ST: Adamalysins in biology and disease. J Pathol 2009;219:277-286.

$\checkmark 15$ Millichip MI, Dallas DJ, Wu E, Dale S, McKie N: The metallo-disintegrin ADAM10 (MADM) from bovine kidney has type IV collagenase activity in vitro. Biochem Biophys Res Commun 1998;245:594-598.
16 Mochizuki S, Okada Y: ADAMs in cancer cell proliferation and progression. Cancer Sci 2007;98:621-628.

17 Herren B, Raines EW, Ross R: Three putative integrin ligands identified in human aortic smooth muscle cells. Ann NY Acad Sci 1997; 811:498-505.

18 Satoh H, Nakamura M, Satoh M, Nakajima T, Izumoto H, Maesawa C, et al: Expression and localization of tumour necrosis factoralpha and its converting enzyme in human abdominal aortic aneurysm. Clin Sci (Lond) 2004;106:301-306.

19 Edwards DR, Handsley MM, Pennington CJ The ADAM metalloproteinases. Mol Aspects Med 2008;29:258-289.

20 Murphy G: Regulation of the proteolytic disintegrin metalloproteinases, the 'Sheddases'. Semin Cell Dev Biol 2009;20:138-145.

21 World Medical Association Declaration of Helsinki: Recommendations guiding physicians in biomedical research involving hu man subjects. Cardiovasc Res 1997;35:2-3.

22 Choke E, Cockerill G, Wilson, Sayed S, Dawson J, Loftus I, et al: A review of biological factors implicated in abdominal aortic aneurysm rupture. Eur J Vasc Endovasc Surg 2005;30:227-244.

23 Thompson RW, Baxter BT: MMP inhibition in abdominal aortic aneurysms: rationale for a prospective randomized clinical trial. Ann NY Acad Sci 1999;878:159-178.
24 Oksala N, Levula M, Airla N, Pelto-Huikko M, Ortiz RM, Jaervinen O, et al: ADAM-9, ADAM-15, and ADAM-17 are upregulated in macrophages in advanced human atherosclerotic plaques in aorta and carotid and femoral arteries - Tampere vascular study. Ann Med 2009;41:279-290.

25 Holdt LM, Thiery J, Breslow JL, Teupser D: Increased ADAM17 mRNA expression and activity is associated with atherosclerosis resistance in LDL-receptor deficient mice. Arterioscler Thromb Vasc Biol 2008;28:10971103.

26 Canault M, Leroyer AS, Peiretti F, Lesèche G, Tedgui A, Bonardo B, et al: Microparticles of human atherosclerotic plaques enhance the shedding of the tumor necrosis factor-alpha converting enzyme/ADAM17 substrates, tumor necrosis factor and tumor necrosis factor receptor-1. Am J Pathol 2007;171:17131723.

27 Al-Fakhri N, Wilhelm J, Hahn M, Heidt M, Hehrlein FW, Endisch AM, et al: Increased expression of disintegrin-metalloproteinases ADAM-15 and ADAM-9 following upregulation of integrins alpha5beta 1 and alphavbeta3 in atherosclerosis. J Cell Biochem 2003;89:808-823.

28 Langer H, May AE, Bültmann A, Gawaz M: ADAM 15 is an adhesion receptor for platelet GPIIb-IIIa and induces platelet activation. Thromb Haemost 2005;94:555-561.

-29 Brocker CN, Vasiliou V, Nebert DW: Evolutionary divergence and functions of the ADAM and ADAMTS gene families. Hum Genomics 2009;4:43-55. 